\title{
Architectures, Protocols and Algorithms for 5G Wireless Networks
}

\author{
Ramón Agüero $^{1} \cdot$ Bernd-Ludwig Wenning $^{2} \cdot$ Yasir Zaki $^{3} \cdot$ Andreas Timm-Giel $^{4}$
}

Published online: 23 September 2017

(C) Springer Science+Business Media, LLC 2017

\section{Guest editorial}

Since its initial edition in 2009, the Mobile Networks and Management (MONAMI) conference has aimed at providing a meeting point for researchers and people working on the mobile and wireless networking realm. The rapid advances of the corresponding technologies, together with the increasing demands from the end users and the appearance of new services, are some of the causes of the ever evolving requirements and challenges that need to be tackled. Together with the conference, we started with a series of Special Issues (SI) of the Mobile Networks and Applications Journal in 2010. Our main objective was to gather works tackling some of the most interesting and current research challenges and issues. The call-for-papers of this SI was therefore an outcome of the 8 th edition of the MONAMI conference, which was held at the University of New York, Abu-Dhabi, in October 2016. Thirty-three papers were received and reviewed by at least three independent and reputable referees. As a result of the process, five manuscripts were selected to be included in this SI. We believe that they together provide a very interesting range of topics that are being currently looked at by the scientific community (and industry) within the wide area of mobile networks and their management. The five papers are

Ramón Agüero

ramon@tlmat.unican.es

1 University of Cantabria, Av los Castros $\mathrm{s} / \mathrm{n}$, 39005 Santander, Cantabria, Spain

2 Cork Institute of Technology, Rossa Ave, Bishopstown, Cork, Ireland

3 New York University Abu Dhabi, Saadiyat Marina District, Abu Dhabi, UAE

4 Hamburg University of Technology, Hamburg, Germany indeed tackling different aspects on wireless technologies, with a clear focus on next generation mobile networks (5G).

We start the SI with the paper entitled "The Network Cloud - Use Cases and Challenges", where M. Hoffmann et al. go beyond previous works on Software Defined Networking (SDN) and Network Function Virtualization (NFV), with a clearer view on the challenges that need to be faced when implementing these components over real mobile networks. The paper argues that most of the existing works on this aspect overlook a number of practical design considerations, recalling that little effort has been made on solving the performance issues that may appear when moving from lab and cloud environments to real networks. They exploit an implementation over commercial off-the-shelf hardware to study $\mathrm{I} / \mathrm{O}$ performance problems, which are identified as one of the biggest challenges of SDN/NFV. The developed framework is used to analyze two complementary use cases for mobile networks: (1) disaggregation of mobile network gateways, and (2) security applications for mobile networks. The paper finishes with an outlook of the most relevant open challenges, which shall also include the design of appropriate migration strategies.

Next, I. Tsai et al. propose, in their paper "Streamlined Paging for Recurrent Mobility Tracking", exploiting predictability of end-user mobility to improve location tracking in mobile networks. The paper arguments that some of the architectural ingredients of 5G, such as ultra-dense network deployments or the C-RAN approach discussed in the previous paper, pose clear challenges to the latency requirement that needs to be guaranteed. They propose reducing the cost (delay) of paging mechanisms to foster the $5 \mathrm{G}$ lightweight connectivity. They combine a generic location management framework (cell masking) with a context mapping process. The former allows a more precise location (down to the cell level), while the latter process relies on information provided 
by the mobile terminals. The system-level based analysis shows that a very relevant reduction of the corresponding overhead can be achieved (especially in the downlink), thanks to the use of the proposed solution.

One of the main drivers behind the rapid increase of mobile data traffic is the massive use of video and streaming services and applications. In fact, video traffic is said to account for $60 \%$ of the overall mobile traffic in 2016. As a consequence, operators and researchers are continuously seeking for solutions to address this situation. In the 3rd paper of this SI, entitled "Improving Video Transmission in Cellular Networks with Cached and Segmented Video Download Algorithms", Ala'a Al-Habashna and Gabriel Wainer exploit device-to-device (D2D) communications, recently introduced in the latest LTE-A specifications. In D2D two mobile nodes can communicate without the need of routing the traffic through the base station, thus bringing interesting capacity gains. The authors propose two algorithms to improve the performance of video transmission over cellular networks. The main idea is to send video file segments to selected users, who will afterwards aid the network infrastructure, by sending the cached segments to other users through D2D links. The performance of the two algorithms is first studied in terms of the hit ratio of video caching, comparing the simulation results with an analytical model that is also proposed in the paper. Afterwards, a system level simulation is used to compare the performance of the proposed solutions with a more conventional approach, studying the impact of end-user mobility over the observed results.

Another technical solution for device-to-device communications is the use of different technologies (for instance, WiFi). In the 4th paper of the SI, M. Alawi et al. provide an extensive discussion on the challenges that need to be tackled to exploit Wi-Fi connections to offload cellular traffic in Vehicular Networking scenarios. In their work entitled "Vehicular Wi-Fi Offloading in Heterogeneous Vehicular Networks: Techniques and Challenges", they distinguish two groups of techniques: (1) infrastructure-assisted solutions, where roadside units are used to offload traffic; and (2) opportunistic vehicle-to-vehicle (V2 V) solutions, where direct V2 V links are exploited. The paper introduces a complete taxonomy to classify the existing offloading techniques, and depicts a potential Vehicular Wi-Fi Offloading (VWO) architectural framework. The paper includes a thorough survey of the solutions that have been proposed. It also discusses some issues that are particularly relevant, such as: prediction accuracy, Road-Side Unit deployment, collaboration among vehicles, use of centralized vs. distributed offloading solutions, evaluation methods, etc. The authors identify the need to focus on Quality of Service, and conclude the paper by identifying the most important research directions in this area.
We conclude this SI with the paper "Regional Route Maintenance Protocol Based on Local Pheromone Diffusion for Mobile Ad Hoc Networks", by H. Choi et al. The authors propose the use of a bio-inspired technique (ant pheromone diffusion) as a novel route maintenance solution for ad-hoc networking scenarios. Routing information elements (pheromones) are locally diffused along the shortest path between source and destination nodes, which is later exploited to discover alternative paths. A complete route maintenance protocol is designed and specified, and the authors discuss its finite state machine. An intensive simulation campaign using the OPNET framework is used to compare the behavior of the proposed solution with other approaches, studying different performance indicators. The evaluation also focuses on how the protocol is affected upon changes on the corresponding scenario (scalability, node mobility, etc). The results that are discussed in the paper show that the proposed protocol outperforms more conventional approaches, while keeping the corresponding overhead at a reasonable level.

Ramón Agüero received a degree in Telecommunications Engineering from the University of Cantabria in 2001 and the $\mathrm{PhD}$ in 2008. He is currently an Associate Professor at the Communications Engineering Department at that university. Since 2016 he is also the Head of the Campus Area. He has participated in several collaborative research projects and his research focuses on future network architectures, especially regarding the (wireless) access part of the network. He is also interested on multi-hop (mesh) networks, device-to-device communications, and Network Coding. He has published more than 160 technical papers in such areas and he is a regular TPC member and reviewer on various related conferences and journals.

Bernd-Ludwig Wenning received Dipl.-Ing. and Dr.-Ing. degrees in Electrical Engineering and Information Technology from University of Bremen, Germany, in 2002 and 2009. From 2002 to 2012, he was a researcher at University of Bremen, participating in several projects in the area of communication networks. In 2012, he joined the Nimbus Centre at Cork Institute of Technology, Ireland, as a Research Fellow. Since then, he has been involved in a number of national and European projects around wireless sensor networks. Throughout his research career, Bernd-Ludwig Wenning has published more than 40 papers in conferences and journals.

Yasir Zaki received his M.Sc. and Ph.D. degrees in Communication and Information Technology from the University of Bremen in 2007 and 2012 respectively. He worked at the Communication Networks group (ComNets) of the University of Bremen as a full-time research associate working on numerous European and Industrial projects. In 2013, he joined New York University Abu Dhabi (NYUAD). Dr. Zaki has over 50 publications in the area of cellular communications and wireless network, his main research areas include: future mobile communication, transport congestion control protocols, and improving the Internet/ network performance in developing countries. 
Andreas Timm-Giel was, between 1994 and 1999, group leader at the University of Bremen in the area of mobile and satellite communications and involved in several EU funded projects. After receiving his $\mathrm{PhD}$ in 1999 he moved to MediaMobil GmbH and M2SAT Ltd. as Technical Project Leader and Manager Network Operations. In December 2002 he joined the Communication Networks group at the University of Bremen as senior researcher and lecturer. He was leading several industrial, national and EC funded research projects and from 2006 he was additionally directing the interdisciplinary activity "Adaptive Communications" of TZI (Center of Computing and
Communication Technologies). In November 2009 he was appointed full professor at Hamburg University of Technology (TUHH) and is heading the Institute of Communication Networks. From 2012 to 2015 he was coordinator of the research cluster SOMSED "Self-organizing mobile sensor and data networks" at TUHH and from 2013 to 2014 deputy head of the TUHH's school of electrical engineering, computer science and mathematics. Since October 2014 he is Vice President for Research at TUHH. His research interests are mobile and wireless communications, sensor networks and the Future Internet. 